\title{
Abnormal Hepatic Function and Splenomegaly on the Newly Diagnosed Acute Leukemia Patients
}

\author{
Sharma Poudel B, ${ }^{1}$ Karki L ${ }^{1}$ \\ 'Department of Medicine, NAMS, Bir Hospital, Kathmandu, Nepal.
}

\begin{abstract}
To evaluate the liver function, splenomegaly and related factors in the newly diagnosed acute leukemia patients. One hundred of fifty eight acute leukemia patients admitted in our hospital from March 2003 to April 2006 were studied. The related factors such as peripheral WBC count, bone marrow blasts, peripheral blasts, sex, age, AML, ALL affecting the liver function and splenomegaly were evaluated. Sixty two (39.24\%) patients presented with splenomegaly. Twelve (7.59\%) patients presented with hepatomegaly. Serum ALT was elevated in $54(34.17 \%)$ patients. Similarly, serum AST, GGT, ALP, and Direct bilirubin were elevated in 26 (16.45\%), 32 (20.25\%), $20(12.65 \%)$, and $22(13.92 \%)$ patients, respectively. Low serum albumin was found in $40(25.31 \%)$ patients. PT was prolonged in $62(39.24 \%)$ patients. Statistical study shows that there is a relation between high WBC counts and elevated serum ALT $(\mathrm{P}<0.05)$ and high WBC counts and splenomegaly $(\mathrm{P}<0.05)$. Acute leukemia patients with leukocytosis are more prone to develop abnormal liver function and splenomegaly.
\end{abstract}

Key words: Acute leukemia, Leukocytosis, Splenomegaly.

\section{INTRODUCTION}

The acute leukemias are a heterogenous group of neoplasms arising from transformation of uncommitted or partially committed hematopoietic cells. ${ }^{1}$ These are a malignant disorder of bone marrow in which maturational arrest in blood cell progenitors results in a failure of normal hematopoesis. Acute leukemias are currently classified pragmatically by combination of differentiation (acute myeloid leukemia versus acute lymphoid leukemia), pathogenesis (myelodysplasia related versus de nevo acute myleloid leukemia), and genetic abnormalities [e.g., acute promyelocytic leukemia with $t(15 ; 17)]$. Obtaining accurate, morphologic, cytochemical, immunophenotypic, and genetic data are critical for guiding treatment decisions.

Hepatomegaly, splenomegaly, and elevation of hepatic enzymes that might be caused by infiltration of leukemia cells are seen in some cases of leukemia. These may result from such conditions as viral hepatitis, liver toxicity due to drugs and leukemic liver infiltration. However, severe liver failure is rarely observed especially at the onset of leukemia and it greatly complicates the initial therapy because most induction agents are metabolized by liver.

\section{MATERIAL AND METHODS}

A retrospective clinical study was done on newly diagnosed acute leukemia patients admitted in hematology ward of the first affiliated hospital of China medical University from March 2003 to April 2006. The diagnosis of acute leukemia was established by bone marrow examination and bone marrow biopsy. Acute myeloid leukemia (AML) and acute lymphoid leukemia (ALL) were

\author{
Correspondence: \\ Dr. Bishesh Sharma Poudyal \\ Department of Medicine \\ NAMS, Bir hospital, Kathmandu, Nepal. \\ Email: drbishesh78@hotmail.com
}


classified according to French American and British (FAB) classification. ${ }^{2}$ Serum alanine aminotransferase (ALT), aspartate aminotransferase (AST), glutamyl transpeptidase, alkaline phosphatase (ALP), albumin (ALB), total protein and prothrombin time (PT) were measured on 24 factor automated chemical analyzer using standard reagents. Ultrasound examination and Computed tomography (CT) scans of liver and spleen were performed to assess hepatosplenomegaly.

Hepatitis B surface antigen (HBs-Ag), hepatitis B surface antibody (anti-HBs), hepatitis B e antigen( $\mathrm{HBe}-\mathrm{Ag}$ ), hepatitis $B$ e antibody (anti-HBe), hepatitis B core antibody (Anti-HBc-lgM, IgG), hepatitis delta antibody (Anti-D), hepatitis A virus antibody (Anti HAV IgM, IgG) and the Human Immunodefiency Virus Antibody (Anti HIV) were all assayed with a second generation enzyme-linked immunosorbent assay (ELISA). The hepatitis C virus antibody was assayed using a second generation ELISA assay. Patients with viral hepatitis were excluded from study. Similarly, the patients with history of alcohol intake, previous intake of hepatotoxic drugs, history of blood transfusion, family history of hepatic disease, history of autoimmune hepatitis, history of hepatomegaly and splenomegaly, history of protein loosing enteropathy, and nephrotic syndrome were excluded from the study.

One hundred and fifty eight patients were found to be eligible for our study, patients ranged in age from 14 to 85 years with mean 42.67 years, median 45 years and standard deviation 17.72 . Eighty four $(53.16 \%)$ were male patients and $74(46.83 \%)$ were female patients. One hundred and twelve $(70.88 \%)$ were $A M L$ patients and 46 (29.11) were ALL patients.

One hundred and fifty eight patients were analyzed with correlation method of kendall's tau_by using Statistical Package for Social Sciences verrsion (SPSS) 11.5. The related factor such as peripheral WBC count, bone marrow blast, peripheral blast, sex, age, AML, ALL affecting the liver function and spleen infiltration were evaluated.

\section{RESULTS}

One hundred and fifty eight acute leukemia patients were studied patients for our study, patients ranged in age from 14 to 85 years with mean 42.67 years, median 45 years and standard deviation 17.72. Eighty four (53.16\%) were male patients and seventy four $(46.83 \%)$ were female patients. One hundred and twelve (70.88\%) were AML patients and 46 (29.11) were ALL patients.

Fifty four patients $(37.17 \%)$ were found to have increased serum alanine trasnferase. Twenty eight were AML
Patients, twenty six were ALL patients. Male patients were twenty two and female patients were thirty two (Table 1). Alanine transferase (ALT) was elevated in the patients presenting with high WBC counts regardless of $A M L$ or ALL $(P<0.05)$. The relationship between serum ALT and sex of patient, age of patients, bone marrow blasts, peripheral blasts could not be established $(P>0.05)$.

Twenty six (16.46\%) patients were found to have increased serum asparatate transferase. Twelve were AML patients, fourteen were all patients. Male patients were twelve and female patients were fourteen. The relationship between serum asparatate transaminase (AST) and sex, age of the patient, AML, ALL, peripheral blasts, bone marrow blasts, peripheral WBC could not be established $(P>0.05)$

Serum gamma glutamyl transpeptidase (GGT) and alkaline phosphatase (ALP) was elevated in patients with high bone marrow blasts regardless of AML or ALL $(P<0.05)$. The relationship between serum GGT and age, sex of the patients, peripheral WBC counts, peripheral blast could not be established $(P>0.05)$. Similarly the relationship between serum ALP and other variables could not be established ( $P>0.05)$.

Twenty two patients $(13.92 \%)$ were found to have increased direct bilirubin. Eighteen were AML patients. Four were ALL patients. Men were sixteen patients and female were six patients. The relationship between serum bilirubin (direct and indirect) and age, sex, peripheral WBC count, bone marrow blasts, peripheral blasts, AML, ALL could not be established ( $P>0.05)$.

Forty patients $(25.31 \%$ ) were found to have decreased serum albumin. Thirty were AML patients, ten were ALL patients. Twenty six were male patients and fourteen were female patients. There was decrease in serum albumin level with increasing age $(P>0.05)$.

Sixty two patients (39.24\%) were found to have increased prothrombin time. Prothrombin time was found to be elevated in $A M L$ patients $(P<0.05)$. The relationship between PT and other variables could not be established $(P>0.05)$.

Sixty two (39.24\%) patients were found to have with splenomegaly. Fourty were AML patients, twenty two were ALL patients (Table 2). Thirty six were male patients, twenty six were female patients. Splenomegaly was found in patients with leucocytosis regardless of AML 
Sharma et al. Abnormal Hepatic Function and Splenomegaly on the Newly Diagnosed Acute Leukemia Patients

or ALL $(\mathrm{P}<0.05)$. The relationship between splenomegaly and other variables could not be established $(P>0.05)$ Twelve patients were found to have hepatomegaly. Ten were AML patient, two were ALL patients. All were male patients. Hepatomegaly was common in male than female histopathological findings in a series of autopies on 110 patients suffering from leukemia and lymphoma, they showed that 10 to 25 untreated patients had neoplastic infiltration. ${ }^{8}$ The clinical and pathological findings in four cases with hepatic failure have been reported. ${ }^{7}$ It is

\begin{tabular}{|c|c|c|c|c|c|c|c|c|c|c|c|c|}
\hline \multirow{2}{*}{$\begin{array}{l}\text { Patients } \\
\text { with } \\
\text { raised } \\
\text { ALT }\end{array}$} & \multirow{2}{*}{ AML } & \multirow{2}{*}{ ALL } & \multirow{2}{*}{ Male } & \multirow{2}{*}{ Female } & \multicolumn{2}{|c|}{ ALT (U/L) } & \multicolumn{2}{|c|}{ WBC (10X9) } & \multicolumn{2}{|c|}{ Marrow blast* } & \multicolumn{2}{|c|}{ Peripheral blast* } \\
\hline & & & & & Range & Median & Range & Median & Range & Median & Range & Median \\
\hline 54 & 28 & 26 & 22 & 32 & 44-713 & 89 & $0.6-213$ & 14 & $25.9-96.4$ & 80 & $0-97$ & 42.5 \\
\hline
\end{tabular}

* in Percent

patients $(P<0.05)$. The relation ship between hepatomegaly and other variables could not be established.

\section{DISCUSSION}

Many authors have reported adult patients with hematological malignancies presenting with fulminant hepatic failure. ${ }^{3,4}$ Zafrani et al, reported four adults with acute monocytic leukemia, acute phase of chronic myeloid leukemia or lymphoma presenting with fulminant hepatic failure. ${ }^{5}$ Harrison et al, reported three patients with metastatic liver disease who presented with clinical course compatible with fulminant hepatic failure. ${ }^{6}$ When publication were reviewed and analyzed, we found anectodal case reports presenting with liver dysfunction and acute leukemia showing spontaneous improvement after initiation of induction therapy. Our study showed patients presenting with abnormal liver function associated with acute leukemia has minimum four weeks history of fatigue and anorexia.

Liver biopsy can be useful and is indicated unless coagulation is abnormal or post hepatic obstruction is suspected. ${ }^{7}$ In our study post hepatic obstruction was ruled out with thorough examination with ultrasonography and CT scans. Coagulation profile although found normal, low platelet count and risk of infection were considered hazard for liver biopsy. Scheinber et al, reported suggested that malignant hematological disease with rapid cellular growth may present as fulminant hepatic failure. Our study showed deranged liver function gets worsen if appropriate treatment of leukemia is delayed. In order to avoid rapidly fatal outcomes secondary to liver failure, early recognization of these malignancies is necessary so as to assure prompt administration of appropriate chemotherapy. ${ }^{5}$ Comeau TB et al, reported patient who developed worsening liver function test results within the first few days of diagnosis. A transvenous liver biopsy was performed, revealing massive lymphoblast infiltration. ${ }^{9}$ Various authors have reported acute leukemia presenting with abnormal liver function. ${ }^{10-16}$

In our study we thought that liver involvement with abnormal liver function was due to leukemic cell infiltration. Although this finding could not be supported by pathological diagnosis, we excluded all other possible causes of abnormal liver function with extensive laboratory examination. Most of the studies have not shown the relation ship between leucocytosis and abnormal liver function. Fingold et al, published thirteen cases of acute leukemia with abnormal liver function who presented with low WBC counts. ${ }^{19}$ Our study was able to show the relation ship between leucocytosis and alaline transferase $(A L T)$ regardless of acute myeloid leukemia or acute lymphoblastic leukemia. ALT is found primarily

Table 2. Types of acute leukemia, sex distribution, WBC, marrow blasts, peripheral blasts in patient with splenomegaly

WBC $10 \times 9$ MARROW BLAST* PERIPHERAL BLAST*

Splenomegaly AML ALL Male Female

Range Median Range Median Range Median

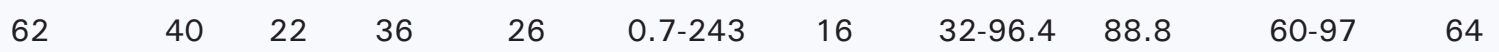

* in percent 
in liver. The aminotransferases are normally present in the serum in low concentration. These enzymes are released into the blood in greater amounts when there is damage to the liver cell membrane resulting in increase permeability. ${ }^{17}$ Simon H.C Anderson et al, also showed similar relationship in their study. ${ }^{10}$

The activation of ALP and gamma GGT are usually elevated in cholestatis. In the absence of jaundice or elevated aminotransferases, an elevated ALP of liver origin often, but not always, suggests early cholestatis and, less often, hepatic infiltration by tumor or granulomata. ${ }^{17}$ In our study increase in serum ALP and gamma GGT were also associated with elevated ALT in most of the cases, this also suggests the elevated ALP and GGT are due to leukemic infiltration. Gall bladder infiltration by leukemic cell is rare. ${ }^{18}$ Liver biopsy in leukemic infiltrated liver showed diffusely necrotic hepatocytes and scattered microsteatosis and extensive portal-portal bands of collapse with loss of parenchyma. ${ }^{19}$ Goor, Yoav et all, published a paper on acute myeloid leukemia presenting as obstructive jaundice. ${ }^{20}$ We strongly believe that the elevated ALP and gamma GGT are due to infiltration of leukemic cells and obstruction of biliary tracts. Our study showed relationship between elevated ALP and GGT with high bone marrow blasts. Milton-J Finegold has reported a case presenting with elevated gamma GGT and ALP with high bone marrow blasts $(98 \%) .^{19}$

With exception of factor VIII, the blood clotting factors are made exclusively in hepatocytes. Measurement of the clotting factors is the single best measure of hepatic synthetic function and helpful in both the diagnosis and assessing the prognosis of acute parenchyma liver disease. ${ }^{17}$ Our study showed prolonged PT in patients with acute myeloid leukemia. Although the patients had prolonged PT, laboratory evidence of other coagulation abnormalities was not present. The standard citrated tube used for coagulation studies contains fixed quantity of anticoagulant for a fixed volume of blood. In leukemia, there is relative reduction of plasma due to excess blast, meaning there will be excess anticoagulant for volume of blood and it may results in falsely prolonged coagulation test.

The spleen functions as a filter of the circulating blood, coordinator of immune response, and a reservoir for circulating cells and platelets. Additionally, the spleen may have number of other responsibilities, including hematopoesis, hemoglobin degradation and iron recovery, and plasma volume regulation. The size of the spleen varies with age as well as immunologic and nutritional status. Pathologic states associated with splenic hematopoiesis include myeloid metaplasia. ${ }^{21}$ However, the production of the cells by spleen under these abnormal conditions results not from the reactivation of fetal stem cell but from displaced bone marrow cells that take up residence in the confines of the organ. ${ }^{22}$ In our study splenomegaly was found in patients presenting with high peripheral WBC counts, similar relation was shown in the other study. ${ }^{23}$

\section{CONCLUSION}

Chemotherapy used in treatment of acute leukemia are also hepatotoxic, our study shows leukemia itself can damage the liver parenchymal cell. While treating the patients with acute leukemia with abnormal hepatic function early recognization of leukemic infiltration is necessary so as to assure prompt administration of appropriate chemotherapy. Although other authors have reported case of acute leukemia presenting with abnormal liver function and splenomegaly their studies was limited to few patients. In conclusion, our study illustrates that acute leukemia patients with leukocytosis are more prone to develop abnormal liver function and splenomegaly.

\section{REFERENCES}

1. Hasse D, Ferring-Buske M, Konemann S, Fonatsh C, Troff C, Veerbek $W$ et al. Evidence for malignant transformation in acute leukemia at the level of early hematopoetic stem cells by cytogenetic analysis of CD34+ subpopulation. Blood 1995; 86(8):2906-2912.

2. Bennet JM, Catovsky D, Daniel MT. Proposals for the classification of the acute leukemias. Brit. J. Haemat 1976;33: 349.

3. Conway EE, Santorineou M, Mitsudo S. Fulminant hepatic failure in a child with acute lymphoblastic leukemia. J PediatrGastroenterol Nutr 1992;15:194-7.

4. Devictor D, Tahiri C, Fabre M, Mielot F, Dussaix E. Early
pre-B acute lymphoblastic leukemia presenting with fulminant liver failure. J Pediatr Gastroenterol Nutr 1996;22:103-106.

5. Zafrani ES, Leclercq B, Vernant JP, Pinaudeau Y, Chomette G, Dhumeaux D. Massive blastic infiltration of liver: a cause of fulminant hepatic failure. Hepatology 1983;3:428-32.

6. Harrison HB, Middleton HM, Crosby JH, Dasher MN Jr. Fulminant hepatic failure: An unusual presentation of metastatic liver disease. Gastroenterology 1981;80:820-825.

7. Ronald H, Leslie S, Edward JB. Hematology basic principles and practice. 3rd edition. London: Churchill Livingstone; 2000. p.1089-1105.

8. Scheimberg IB, Pollock DJ, Collins PW, Doran HM, Newland 
AC, van der Walt JD. Pathology of liver in leukemia and lymphoma. A study of 110 autopies. Histopathology 1995;26: 311-21.

9. Comeau TB, Phillips DL. Chemotherapy dosing with elevated liver function test results in acute leukemia. Ann Pharmacother. 2005 Oct;39(10):1752-4. Epub 2005 Aug 30.

10. Anderson SH, Richardson P, Wendon J, Pagliuca A, Portmann B. Acute liver failure as the initial manifestation of acute leukemia. Liver 2001;21(4):287.

11. Handa H, Motohashi S, Isozumi K, Komatsumoto S, Nara M. CD7+ and CD 56+ Myeloid/Natural killer cell precursor acute leukemia treated with Idarubicin and Cytosine arabinoside. Acta Hematol 2002;108:47-52.

12. Kuronda N, Mizobuchi M, Shimamura Y, Daibata M, Miyoshi I, Ohara $\mathrm{M}$, et al. Bridging necrosis and reticulin bridging fibrosis induced by intrahepatic involvement of acute biphenotypic leukemia Acta Pathologica microbiologica et Immunologica. Scandinavica (APMIS) 2006;114(12):908.

13. Takamatsu T. Preferential infiltration of liver sinusoids in acute lymphoblastic leukemia. Rinso ketsueki. Japan society of clinical hematology 2005;42(12):1181-86.

14. Litten JB, Rodríguez MM, Maniaci V. Acute lymphoblastic leukemia presenting in fulminant hepatic failure. Pediatric blood and cancer 2005;47(6):842-5.

15. Scolyer RA, Brun M, D'Rozario J, Webb M. Acute basophilic leukemia presenting with abnormal liver function tests and the absence of the blast cells in the peripheral blood. Pathology 2000;32(1):52-55.

16. Kawamura E, Habu D, Kurooka H, Hayashi T, Oe A, Kotani $\mathrm{J}$ et al. Adult $\mathrm{T}$ cell leukemia blast crisis in a patient with liver failure. Indian journal of gastroenterology 2006;25(1):49.

17. Eugene B, Anthony SB, Dennis LK, Stephen LH, Dan LL, Larry J. Harrison principles of internal medicine.15th ed. New York: McGraw-Hill; 2001. p.1711-15.

18. Shimizu T, Tajiri T, Akimaru K, Arima Y, Yokomuro S, Yoshida $\mathrm{H}$ et al. Cholecystitis caused by infiltration of immature myeloid cells. J Nippon medical school 2006;73:97-100.

19. Milton J. Finegold. Childhood leukemia presenting with acute hepatic failure. Liver pathology speciality conference: Annual meeting; 2005.

20. Goor Y, Goor O, Michalewitcz R, Cabili S. Acute myeloid leukemia presenting as obstructive jaundice. Journal of clinical gastroenterology2002;34(4):485-6.

21. Robins SL, Cotran RS, Kumar V. Robin's pathologic basis of disease. 5th ed. Philadelphia: WB Saunders; 1994. P.629-72.

22. Wilkins BS, Green A, Wild AE. Extramedullary hemopoeisis in fetal and adult human spleen: a quantatative immunohistological study. Histopathology 1994;24:241-7.

23. O'reilly RA. Splenomegaly in 2,505 patients in a large university medical center from 1913 to 1995. 1913 to 1962: 2,056 patients. West J med 1998 Aug;169(2):78-87. 